\title{
La Détente y el Sistema Internacional
}

\author{
INTRODUCCIÓN
}

El propósito de este trabajo es ayudar a clarificar el significado de uno de los términinos más utílizados en el lenguaje de las Relaciones Internacionales después de "Guerra fría": la "distensión" o "détente".

Tratándose de un fenómeno polivalente para los distintos tipos de observadores políticos, el aporte académico podría consistir en establecer con rigurosidad el contenido específico de este concepto, y su virtual utilidad como herramienta para el estudio de las Relaciones Internacionales en la década presente.

Sin un conocimiento sobre el actual "estado de la cuestión", las discusiones sobre el tema pueden situarse en un nivel puramente evaluativo o de interpretación general, saltando una etapa previa de carácter descriptivo, que puede servir, al menos, para uniformar el lenguaje entre los estudiosos de los problemas de la politica mundial.

El periodo seleccionado se refiere a los años 1968-1974 que parecen ser los años más representativos de esta nueva etapa de las Relaciones Internacionales. La naturaleza de este trabajo es más bien exploratoria y descriptiva. Es decir, se pretende entregar una información básica e introductoria sobre la materia.

Mediante este esfuerzo se intentará también identificar el espectro de alternativas que pueden estar presentes en el horizonte diplomático del Tercer Mundo y de América Latina dentro del nuevo ordenamiento internacional.

En relación a las fuentes, se utilizará, en primer lugar, una revisión de los hechos más destacados que han sucedido desde 1969 a 1974. Esta serie de acontecimientos ha alterado no sólo las reglas del juego entre las super-potencias, y entre éstas y sus aliados, sino que, además, ha modificado el contenido y la dirección de las políticas exteriores de los países neutrales y no-alineados.

La segunda fuente de información son las declaraciones oficiales y documentos emanados de los gobiernos que con mayor frecuencia apuestan a la distensión en el tablero de la política mundial.

La opinión de los grupos de interés que intervienen en las decisiones de política exterior de los diferentes gobiernos ha sido recolectada mediante el estudio de las sesiones especiales sobre la détente 
que ha realizado el parlamento estadounidense, y por medio de varios documentos oficiales del Partido Comunista Soviético, de la asamblea de la R. P. China y de los diversos parlamentos europeos.

Finalmente, por medio de la lectura cuidadosa de la bibliografía que se adjunta, se obtienen las opiniones calificadas de un grupo de expertos en asuntos internacionales que han analizado este fenómeno durante el último tiempo.

De esta manera se ha combinado tres tipos de información que por su carácter primario y secundario pueden hacer más consistente las observaciones de este trabajo.

\section{¿QUÉ ES LA DISTENSIÓN?}

a) Según el profesor Walter Clemens, de la Universidad de Harvard, esta palabra aparece en el idioma inglés, a comienzos del siglo xx. En distintos idiomas, la palabra conserva el significado proveniente de su raíz etimológica, es decir, el verbo "destendre" del francés antiguo, que significa "relajar", "suavizar"."

b) Sin embargo, este supuesto aliviamiento de las tensiones y fricciones no tiene el mismo significado para las super-potencias y para las potencias regionales, especialmente los países europeos, que aparecen como los actores más comprometidos en esta nueva forma de equilibrio de poder. "Para las super-potencias el concepto de la détente lleva implícito la mantención del status quo. Es utilizada en el sentido de ausencia de tensiones y amenazas en las relaciones "Oriente-Occidente" o para señalar una atmósfera en la cual la parte contraria está dispuesta a hacer concesiones..." Por el contrario "los europeos han llegado a concebir la détente como una diplomacia flexible, con un flujo creciente de relaciones europeas, y como la oportunidad para cambiar el rostro político de Europa. Ha sido acompañada por cambios de visión sobre problemas tales como las fuentes de la amenaza y las fuentes de la seguridad". ${ }^{2}$

Estas diferentes connotaciones del concepto "distensión" fortalecen sin duda nuestra búsqueda de mayor esclarecimiento y señala el peligro real que existe cuando un concepto se mitifica o se vincula a una persona o a un gobierno determinado.

${ }^{1}$ Ver Clemens C. Walter, The impact of Détente on Chinese and Soviet Com munism, Journal of International Affairs, vol. 28, No 2, 1974. "The Meaning of Détente", State Department Pub., 8766, June 1974.

${ }^{2}$ Ver Laszlo Hadik, "The Process of Détente in Europe", Orbis, vol, XIII, Winter 1970 , No 4, p. 1008. 
c) El tratamiento más ilustrativo de este problema parece ser la contrastación de este concepto con otra forma de equilibrio de postguerra, conocida como la "guerra fría".

Arthur Schlesinger, en su artículo sobre los orígenes de la "guerra fría", define este último proceso como: "un antagonismo mortal, nacido al comienzo de la Segunda Guerra Mundial, entre dos bloques definitivamente hostiles, uno bajo el liderazgo de la Unión Soviética, el otro dirigido por los Estados Unidos". 3

E1 período de postguerra aparece basáado en una completa rivalidad estratégica y en un completo desacuerdo sobre la organización dé ia paz. Las profundas diferencias ideológicas entre los bloques le dieron a este conflicto un matiz semi-religioso, semejante a los enfrentamientos anteriores al Tratado de Westfalia en 1648. Desde e1 punto de vista ideológico, esta rivalidad puede ser ilustrada con la actuación de John Foster Dulles. En el plano estratégico hay autores que sostienen que la primera bomba atómica no fue lanzada por la urgente necesidad militar de poner fin a la guerra, sino que por el efecto disuasivo hacia el "avance" de la Unión Soviética.4

Sin apoyar totalmente la tesis anterior, es interesante comprobar que el "equilibrio del terror" logra mantener al planeta libre de una tercera guerra mundial, al costo de una carrera armamentista en escalonamiento progresivo.

Sin embargo las super-potencias nucleares y las potencias que pronto ingresarian a este exclusivo club, comienzan a cuestionar la utilidad real de su poder disuasivo y la vigencia de la guerra fría. Paulatinamente, la "Realpolitik" soviética, la "Ostpolitik" alemana y las declaraciones del presidente Kennedy quien reconoce "que no puede haber una solución americana para todos los problemas del mundo" (1961) inician una revisión paulatina del status quo vigente hasta llegar a su modificación, dando lugar al fenómeno de la distensión.

De esta manera, la guerra fría se sostuvo por medio de un latente y a veces manifiesto antagonismo de dos polos irreconciliables representados por los Estados Unidos y la Unión Soviética.

Mediante esta comparación de dos formas de equilibrio del poder dentro del sistema internacional de postguerra, se puede identificar cuál es el concepto de la distensión.

Este fenómeno puede entenderse como resultante no sólo de un

${ }^{8}$ A. M. Schlesinger, "Origins of the Cold War", Foreign Affairs, October 1967.

"Dr. Alperovitz citado por Adam Ulam "On Modern History-Re-reading the Cold War", Hearings before the Subcommittee on Europe: The Cold War: Origins and Developments. U.S. Printing Office, Washington, D.C., 1971, p. 23. 
cambio táctico de la política exterior entre las super-potencias. En efecto, dentro del sistema internacional se van acumulando una serie de acontecimientos, tales como la paridad nuclear y militar entre los EE. UU. y la Unión Soviética; el cisma en el interior del bloque socialista y la formación de una estructura de poder policéntrica en reemplazo de la estructura mundial bipolar.

Esta nueva estructura, incorporará en su seno a la Europa Occidental recuperada de la Guerra, al Japón y a la R. P. China. Finalmente, los países exportadores de petróleo, también han manifestado su vocación por interferir en Ios asuntos mundiales mediante la nueva arma del petróleo.

En resumen, para responder la pregunta ¿Qué es la đistensióñ̂́, hemos recorrido al menos tres caminos:

1. - Los aspectos etimológicos del término;

2. - La comparación del doble significado del concepto para los super-poderes y Europa, y

3. - Contrastación de los términos "guerra fría" y "distensión".

d) Sin embargo, existe una cuarta alternativa para responder a nuestra interrogante. Se trata de entender la distensión dentro de un espectro o un continuum. Este expediente heurístico lo utilizan algunos especialistas y parece bastante didáctico. En concreto, la distensión se concibe como un punto de un espectro lógico de relaciones en las cuales la distensión aumenta o disminuye.

$\mathrm{Si}$ las tensiones aumentan, por ejemplo, las partes en conflicto pasan desde la posición de guerra fría a la de enfrentamiento directo, la distensión disminuye. Siguiendo este camino, se observa que desde 1945 a 1973 , las situaciones que más tipificaron a una situación de guerra fría fueron, en orden de prioridad: la Guerra de Corea (1950-51); la creación de la OTAN (1949); el bloqueo de Berlín (1948) y, posteriormente, en igual orden: la crisis de los Misiles (1962) y la confrontación de Berlín (1960-62).

Por otro lado, las situaciones que más se aproximan al concepto de distensión son, de menor a mayor importancia: Los tratados de No-Proliferación (1968); SALT (1969); y finalmente las diversas reuniones en la Cumbre. Los orígenes de estos acontecimientos se remontan a la reunión en la Cumbre en Ginebra (1955); la reunión de Camp David (1959) y al "espíritu de Moscú" (1964).

Aplicando la "teoría del péndulo" a estas observaciones, se podría decir que a partir de la "crisis de los Misiles" la dirección de los acontecimientos se distancian del polo de la guerra fría pasando por zonas de "entente" y "contención" hasta llegar al extremo opuesto de la "distension", caracterizado por las reuniones en la cumbre 
entre los jerareas de la Unión Soviética y Estados Unidos y, posteriormente, entre estos atimos y los de la R. P. China. ${ }^{5}$

e) Con estas consideraciones, la distensión puede definirse como un juego en el cual los participantes pueden perder a ganar, colaborar o enfrentarse o, por último, aliarse con otros jugadores de modo de complementar su propio juego y, así permitir relaciones de intercambio que no son puramente conflictivas o armoniosas. Este tipo de juego se asemeja a lo que Karl Deutsch denomina un "juego de suma-variable" o de motivo mixto. En estos juegos los jugadores no sólo ganañ algo competitivamente uno de otro, sino que también tienen la probabilidad de ganar o perder colectivamente. Son juegos de competición, en la medida en que tratan de ganarse uno a otro; pero también son juegos de coordinación, puesto que los jugadores ganarán o perderán también conjuntamente. ${ }^{6}$

Al contrastar este tipo de juego con el juego político de la guerra fría se pueden observar diferencias significativas. Según Schlesinger, la guerra fría se asemejaba a un duelo en el cual la pérdida de un jugador significaba su derrota $y$, por lo tanto, la victoria total de su enemigo. Es decir, lo que es bueno para un jugador, es necesariamente malo para su adversario y viceversa. En el lenguaje de la teoría de los juegos estaríamos en la presencia de un juego "sumacero" o de "suma-fija". La guerra fría entonces, se podría resumir -según algunos observadores- de la siguiente manera:

"Todo lo que es bueno para el Oeste, o incluso aceptable para él, debe ser obviamente malo para el comunismo, y todo lo que es bueno para el comunismo, o incluso meramente aceptable para él, debe ser automáticamente malo para Estados Unidos. Cualquier paso que se dé hacia la moderación, la adaptación mutua o el compromiso entre EE. UU. y la URSS o entre EE. UU. y China Comunista, en cualquier cuestión, según piensan los "verdaderos creyentes" de ambos bandos en la guerra fría no es nada más que un fútil apaciguamiento de un enemigo insaciable y un sacrificio traicionero del interés de la propia nación". ${ }^{7}$

Ahora bien, al examinar la política realista (realpolitik) que practican las dos super-potencias observamos que éstas apuntan a la mantención del sistema internacional mediante soluciones de compromiso, intercambio mutuo y aceptación del principio de la "guerra

"William Gamson y A. Modigliani, "Untangling the Cold War", Little Brown \& Co., Boston, 1971, pp. 196-199 y Clemens, op. cit., p. 36. "Ver gráfico explicatorio".

${ }^{\circ}$ Karl W. Deutsch, "El Análisis de las Relaciones Internacionales", Ed. Paidós, Buenos Aircs, 1970, pp. 143-144.

IIbid., p. I4I. 
limitada". Henry Kissinger, uno de los precursores del concepto, sostiene que si bien la guerra limitada no era preferible a la paz, sí lo era a la rendición o a la guerfa total. Según él, la idea de la represalia masiva habfa perdido casi toda su "credibilidad" para no referimos a s: "utilidad". El expediente de guerra limitada, en cambio, al menos permitía soluciones de compromiso, derrotas o victorias limitadas y situaciones de empate. ${ }^{8}$

De este modo se observa que aunque las diferencias cruciales persisten entre las superpotencias, en el esquema de distensión, estas incompatibilidades se regulan al máximo de modo de evitar conflictos mayores y mantener el flujo de intercambios ya sean de carácter político, económico o culturat.

Este fenómeno del "intercambio" parece ser un elemento distintivo del proceso de la distensión. ${ }^{\circ}$ En este contexto la siguiente definición de "détente" parece respaldar la observación anterior: "Détente significa, por lo general, la asociación del gobierno y los empresarios norteamericanos en el relajamiento de la tensión política permitiendo, simultáneamente, que se desarrolle un modelo de intercambio comercial "normal" entre los Estados Unidos y la Unión Soviética... En su contexto histórico la dinámica de esta asociación marca el término efectivo de la guerra fría que comenzó cerca de treinta años atrás". ${ }^{10}$

Por cierto que esta definición refleja la opinión de un grupo de interés. Sin embargo, a lo largo del documento que contiene las audiencias sobre la détente ante el congreso norteamericano, aparecen distintas corrientes de opinión sobre el fenómeno de la distensión. En concreto, surge una nueva percepción del rol cle la Unión Soviética en los asuntos internacionales.

En el plano ideológico, esta nueva visión se ve reflejada en "una disminución del fervor de más de medio siglo de preocupación norteamericana... acerca de implicaciones del comunismo soviético (para los EE. UU.). Esta preocupación todavía no ha cesado completamente. Pero su intensidad... hoy en día alcanza su nivel más bajo desde la Segunda Guerra Mundial".11

"Sobre el concepto de "guerra limitada", scgún Kissinger, ver Stephen F. Graubard, "Kissinger: Retrato de una mente", Ed. Dopesa, Barcelona, 1973, capitulos 3 y 4.

${ }^{\circ} \mathrm{Ej}$. Acuerdos de intercambio de abril, agosto, noviembre de 1971; febrero y julio de 1972; marzo y junio de 1973; febrexo, abril y mayo de 1974, en cl campo comercial, científico, tecnológico, cultural.

${ }^{10}$ Benjamín S. Rosenthal en "Détente", Hearings beforc the Subcommittee

on Europe of the Committee on Forcign affairs of the House of Representatives U.S. Government Printing Office, Washington, 1974, p. VII. Además, ver "Déten$t e$ ", Hearings before the Committee on Foreign Relations of the U.S. Senate. U.S. Government Printing Office, Washington, D.C., 1975.

${ }^{11}$ Ibid., P. VII. 
Pese a la distensión, entonces, subsiste un grado considerable de "hostilidad ideológica” entre Washington y Moscú.

LOS FUNDAMENTOS DE LA DISTENSIÓN

Después de este breve recorrido para establecer el significado del concepto "distensión" y sus diferencias con otras formas de equilibrio de poder en el sistema internacional, parece conveniente analizar cuáles son los distintos motivos que han desencadenado la serie de hechos registrados entre los años 1968 y 1974.

Por fundamentos de la distensión se entiende aquellos intereses de Estado (raison d'Etat) que ambas potencias tratan de asegurar, promover y coordinar por medio de la distensión. Las condiciones estructurales que en forma acumulativa dieron lugar a este cambio serán analizadas más adelante. Por ahora nos interesa una lectura cuidadosa de cuál es la racionalidad propia del proceso de distensión.

En concreto, existen intereses estratégicos por ambas partes en la détente que podrían condensarse de la siguiente manera: "EE. UU. obtendrá una reducción de las tensiones políticas y, por lo tanto, una disminución del presupuesto militar, y, un mejoramiento de su balanza de pagos; mientras que el bloque oriental obtiene acceso a tecnología avanzada y a créditos e inversiones de capital para mejorar su economía y acelerar el desarrollo de los recursos naturales soviéticos $\mathrm{y}$, lo que es más importante, obtendrá acceso a los grandes stocks de granos norteamericanos que le ayudará a solucionar problemas de abastecimiento y alimentación". ${ }^{12}$

Sin embargo, es conveniente agregar que esta lógica interna de la distensión no puede entenderse al margen de la relación tripolar Moscú-Washington-Pekín. Específicamente, el interés soviético por aumentar el acercamiento con EE. UU., Europa Occidental y Japón obedecería a la intención de prevenir el avance de la República Popular China. Por otra parte, el interés norteamericano principal sería neutralizar los avances soviéticos mediante el mejoramiento de relaciones con Pekín. Paradojalmente China estaría jugando el rol de "balanceador" en este nuevo esquema de equilibrio, mientras que EE. UU. ocuparía un rol análogo en el conflicto chino-soviético.

En resumen, al examinar la racionalidad de la détente, se concluye que existe un grado de unidad global en medio de intereses distintos y a veces contradictorios. Se sabe que hay diferentes expectativas de

"Walter Krause, citado en "Détente", ibid., ver partes I y II. 
beneficios que cada actor trata de obtener. Como fue ilustrado anteriormente, las esperanzas cifradas en este nuevo "modus vivendi" son distintas para las super-potencias y para Europa. Una situación parecida sucede con la República Popular China.

Sin embargo, a pesar de las diferencias en lo particular parece que existiera acuerdo en hacer un "esfuerzo mínimo crítico", al decir de los economistas, para aceptar la détente como un proceso global y globalizante.

Al hablar de una dinámica global, se quiere decir que la distensión no es una táctica con un objetivo excluyente sino una estrategia que genera una totalidad de relaciones, en la esfera de la alta polftica, y en esta característica radicaría uno de los fundamentos que le dan coherencia.

Al respecto, Arthur H. Hartman, secretario adjunto para los asuntos europeos declaraba en la cámara lo siguiente: "La détente no es algo estático, algo que se persigue, un objetivo específico. Es una totalidad de relaciones... Nosotros consideramos que la détente es un proceso y ell un proceso como éste, la totalidad de las relaciones es lo que debe tomarse en cuenta". ${ }^{13}$

En este párrafo Hartman se refiere incluso a un problema moral como el de los derechos humanos en la Unión Soviética, y en otras partes del globo, afirmando que "lo que se haga, o deje de hacer, en relación a los derechos humanos afectará la totalidad de las relaciones y se convertirá en parte del proceso total". ${ }^{14}$

El común denominador en este nuevo entendimiento, al menos al interior de la estructura multipolar del mundo, serfa que este conjunto de relaciones económicas, culturales y políticas acepta el supuesto mínimo que es más conveniente la negociación parcial que el enfrentamiento global.

LA DISTENSIÓN Y EL ESQUEMA PENTAGONAL DEL MUNDO

En la sección anterior se resumieron los elementos que fundamentan la détente como un esquema de coordinación de las políticas exteriores de las grandes potencias.

En esta parte se examinarán, en primer lugar, aquellos cambios en las estructuras que organizan y distribuyen el poder mundial y que tienen una relación causa-efecto con la gestación del nuevo "modus

"'Détenle", op. cit., p. 56.

${ }^{14}$ Ibid., pp. 56-57. 
vivendi" internacional y, en seguida, examinaremos el pensamiento político del Secretario de Estado Henry Kissinger como un elemento importante en el desarrollo del fenómeno de la distensión.

En los mensajes anuales al Congreso norteamericano de 1970 y 1973, el ex presidente Richard Nixon señaló varios elementos que habrían dado origen al cambio en la filosofía y la práctica de la política exterior norteamericana. ${ }^{15}$

En forma resumida se pueden enumerar los siguientes puntos: en primer lugar, se da por terminado el período de postguerra en que EE. UU. era la potencia dominante. En segundo término, se produce la recuperación política y económica de Japón y Europa. En seguida, las nuevas naciones empiezan a participar activamente en los asuntos internacionales con miras a reforzar su independencia nacional. En cuarto lugar, la "unidad monolítica" del bloque socialista se diluye $\mathrm{y}$, además, la República Popular China ingresa al club nuclear. Quinto, la Unión Soviética y Estados Unidos alcanzan una "paridad nuclear". Este empate del poder estratégico es resumido por el propio Nixon: "Desde 1945 a 1949 nosotros éramos la única nación del mundo que poseía un arsenal de bombas atómicas. Desde 1950 a 1966 nosotros teníamos una superioridad estratégica sin contrapesos. Desde 1967 a 1969 mantuvimos una superioridad significativa. Hoy en día, la Unión Soviética posee una fuerza estratégica poderosa y sofisticada casi equivalente a la nuestra" ${ }^{10}$. Finalmente, a partir del discurso presidencial pronunciado el año 1969 en la isla de Guam, se establece la tesis central de la denominada "Doctrina Nixon", que consiste en que "EE. UU. participará en la defensa de los países aliados y amigos, pero no podrá concebir 'todos' los planes, designar 'todos' los programas, ejecutar 'todas' las decisiones y llevar a cabo 'toda' la defensa de las naciones del mundo". ${ }^{17}$

Esta serie de cambios en las estructuras representa una nueva etapa en la evolución de las relaciones internacionales, y es precisamente en este período (1968-1974) cuando la détente cobra una importancia y un ritmo creciente ${ }^{18}$. Una de las causas que explican la curva ascendente del proceso de distensión ha sido la propia concepción del sistema internacional de Henry Kissinger, ex asesor es-

${ }^{16}$ Ver "United States Foreign Policy for the 1970's: a New Strategy for Peace", U.S. Government Printing Office, Washington, 1970. "U.S. Foreign policy for the 1970's: Shaping a Durable Peace", U.S. Government Printing Office, Washington, 1973.

10"U. S. Foreing Policy for the 1970's: a New Strategy for Peace", op. cit., p. 10.

${ }^{17}$ Ibid,, p. 6.

${ }^{18}$ De 108 tratados entre EE. UU. y la URSS desde 1933, 58 de ellos se firmaron entre 1972 y 1974. 
pecial de Nixon en política internacional y actual secretario de Estado del presidente Ford.

El propósito de Kissinger, como se puede comprobar a través de sus principales escritos, es llegar a establecer un orden "legítimo" internacional. Cabe señalar que el uso que Kissinger da al término "legitimidad" no debe confundirse con el de "justicia". La legitimidad, apunta Kissinger, implica "la aceptación del marco del orden internacional por todas las grandes potencias, por lo menos hasta el punto en que ningún Estado esté tan descontento que, como Alemania después del Tratado de Versalles, exprese su insatisfacción en una política exterior revolucionaria. ${ }^{19}$

Por lo tanto, un orden internacional en que las disposiciones básicas son aceptadas por todas las potencias importantes puede ser calificado como "legítimo". Un sistema que tiene una potencia o un grupo de potencias que rechazan las disposiciones o el establecimiento de la estructura interior de los demás Estados - por ejemplo el que caracterizó al período de la guerra fría- es revolucionario. Un orden legítimo no necesariamente hace imposible los conflictos, pero al menos, afirma Kissinger, limita su extensión; pueden producirse guerras pero éstas son libradas en nombre del sistema existente. $^{20}$

E1 objetivo central de la Doctrina Kissinger entonces es pasar de un período revolucionario a un período de estabilidad, como el que surgió del conflicto revolucionario en la Europa de 1822. El artífice principal del restablecimiento del equilibrio en ese entonces fue el ministro austríaco Clemens von Metternich a quien Kissinger define como "un científico de la política, que arreglaba sus combinaciones fríamente y sin emoción, en una época donde la política se conducía cada vez más por causas". ${ }^{21}$

Inspirado en la figura de Metternich, Kissinger busca instaurar un nuevo "período de estabilidad" mejorando las relaciones diplomáticas con la URSS. y la República Popular China. Procura "legitimar" el actual orden internacional haciendo uso preferente del instrumento de la diplomacia"22 que él define como: "el arte de relacionar a los Estados entre si por el consentimiento antes que por el ejercicio de la fuerza, por la presentación de un campo de

\footnotetext{
${ }^{10}$ Henry Kissinger, "Un Mundo Restaurado", Fondo de Cultura Económica, México, 1973, pp. 11-12.

${ }^{30}$ Henry Kissinger, "Armas Nucleares y Politica Internacional", Ed. Ria]p, Madrid, 1958, p. 74.

21"'Un Mundo Restaurado", p. 406.

"No obstante, el ministro norteamericano se ha pronunciado en contra del divorcio entre la fuerza y la diplomacia.
} 
acción que concibe las aspiraciones particulares con un consenso general".28

Como podemos apreciar entonces, el proceso de distensión se encuadra perfectamente con el pensamiento "kissingeriano" que aparece expresado, en forma concreta, en la denominada "concepción pentagonal del mundo". De acuerdo con esta concepción, la paz mundial descansaría en cinco polos principales de poder, tres super-potencias de influencia planetaria, los Estados Unidos, la Unión Soviética y la República Popular China y dos potencias de influencia regional, los países del Mercado Común Europeo y Japón. Ante esta interpretación cabe plantearse varias interrogantes, por ejemplo: ¿Cuál es el rol del Tercer Mundo en este proceso de distensión? ¿Cuáles serán las consecuencias de la détente para los países en váa de desarrollo?

\section{LA DISTENSIÓN Y EL TERCER MUNDO}

No es de extrañar que los paises subdesarrollados vean en la détente una suerte de entendimiento entre los grandes - a espaldas de los pequeños-, los cuales, una vez resguardado su "frente interno" entre ellos podrian ejercer su hegemonía sobre el resto del mundo. Al respecto, es interesante destacar que los chinos afirman que la lucha del Tercer Mundo ya no es sólo contra el colonialismo y el imperialismo, sino que, además contra lo que denominan el "hegemonismo" de las super-potencias. De allí que sostengan que: "Moscú intentó imponer un 'sistema de seguridad colectiva asiática a los asiáticos, mientras que Washington, una comunidad del hemisferio occidental' a los latinoamericanos... (Pero) los países de Asia, Africa y América Latina están resueltos a administrar sus propios asuntos, y con este propósito establecen y refuerzan las organizaciones regionales que excluyen a las dos super-potencias, y transforman algunas antiguas dominadas por las grandes potencias". ${ }^{24}$

El contenido de la détente parece ser - por tanto- ambivalente: puede convertirse en un instrumento hegemónico o puede servir de marco para la creación de un sistema internacional justo. ${ }^{25}$

Según Henry Kissinger, la concepción general de la détente "se

98"Un Mundo Restaurado", p. 414.

"Ver "Surgimiento del Tercer Mundo y Declinación del Hegemonismo" por el corresponsal Sinjua, Boletín Informativo de la Embajada de la República Popular China, Santiago, 1975.

${ }^{20}$ Ver Gustavo Lagos, "Vigencia de la Détente o Epilafio para la Humanidad"', Mensaje, oct. 74, No 233, pp. 462-463. 
opone a la idea de que haya una 'détente de preferencia', en la que se pacificaría una región mientras en otra se mantendría un conflicto activo"26. Kissinger ha manifestado que "es erróneo hablar de un solo equilibrio de poder, ya que existen varios que tienen que relacionarse entre si. En la esfera militar, existen dos superpotencias. En términos económicos, se cuentan por lo menos cinco agrupaciones principales. Políticamente, han surgido muchos centros más de influencia... nuestro objetivo inmediato ha sido construir una red estable de relaciones que ofrezca la esperanza de librar a la humanidad de los estragos de la guerra. Una comunicación mundial interdependiente no puede tolerar ni la confrontación de gran. des potencias ni la repetición de crisis regionales". ${ }^{27}$

Por lo tanto, la détente, según Kissinger, sería indivisible y, en términos globales, significaría lo siguiente ${ }^{28}$ :

1. - El entendimiento entre los EE. UU. y la URSS. no tiene el propósito de aliviar las tensiones entre ellos para quedar con las manos más libres frente a los paises débiles.

2. - La concepción pentagonal es flexible ya que puede permitir la emergencia de centros de poder adicionales.

3. - La multipolaridad significa una mayor libertad de acción para todos los países.

4. - La détente entre las grandes potencias no es preferencial en el sentido de que no se hace a expensas del resto del mundo sino que es una primera etapa indispensable para evitar la guerra nuclear y para la reorganización del sistema internacional.

Ahora bien, ¿qué ha significado la détente en el caso particular de América Latina?

Un caso especialmente significativo de las consecuencias de la distensión en nuestro continente, ha sido el nuevo rumbo que han tomado las relaciones EE. UU.-Cuba y Cuba-América Latina

La détente, la experiencia de la guerrilla boliviana de 1967, la aparición de regímenes nacionalistas y antiimperialistas en el hemisferio, $y$ otros factores, han motivado un cierto cambio en la política exterior cubana. De allí que hoy en día Cuba apoye al gobierno peruano de Velasco Alvarado, al gobierno de Omar Torrijos en Panamá y al gobierno mexicano de Luis Echeverría. Asimismo, las relaciones han mejorado notablemente con países tales como Argen-

20" El punto de vista de Kissinger sobre la détente y las naciones en desarrollo", Texto Oficial de USIS, Washington, mayo 14, 1974.

${ }^{27}$ Ibid.

${ }^{29}$ Gustavo Lagos, op. cit., p. 462. 
tina, Venezuela y Colombia, con quienes Cuba mantiene relaciones diplomáticas formales. ${ }^{29}$

Por otra parte, se ha producido un lento acercamiento entre Cuba y EE. UU., estimulado por las visitas a la isla de los senadores norteamericanos Javits y Pearl, más el reciente viaje del senador demócrata y ex candidato presidencial George McGovern. Cabe destacar que todos se han pronunciado -al regreso de sus viajes- en favor del levantamiento del "boicot" comercial.

En los hearings del "Great Decisions Program" ante el Comité de Relaciones Exteriores del Senado norteamericano se ha sostenido que la gran mayoría de los ciudadanos norteamericanos apoyaría un cambio en la política exterior estadounidense hacia la isla caribeña. Una encuesta Louis Harris llevada a cabo en marzo de 1973 habría indicado que "un número creciente de norteamericanos, una mayoría nacional, favorece el restablecimiento de relaciones con Cuba". ${ }^{30}$

Recientemente, Ron Nessen, el principal portavoz del presidente Ford ha declarado que: "Estados Unidos ha dicho que no vemos futuro en el antagonismo perpetuo hacia Cuba". Añadiendo que, no obstante, "hay áreas de diferencias significativas entre los dos países". ${ }^{31}$

Sin embargo, aunque la détente entre los Estados Unidos y la Unión Soviética ha contribuido en cierto modo al mejoramiento de relaciones entre EE. UU. y Cuba, si consideramos la situación de América Latina en general, tendríamos que concluir afirmando que han sido los "acontecimientos domésticos" -y no la distensión internacional- los que han resultado más significativos en la determinación de las actividades de los principales grupos políticos latinoamericanos. $^{32}$

Un estudio detallado de la problemática latinoamericana debería incluir un análisis crítico de la nueva ley de Comercio Exterior y del denominado "nuevo diálogo", además de otros acontecimientos nacionales y regionales que no pueden tratarse, en forma rigurosa, en este breve trabajo. Lo que sí interesa destacar es que en la medida que este nuevo "modus vivendi" de las relaciones internacionales

\footnotetext{
${ }^{30}$ En la XVI asamblea de consulta de la OEA (Costa Rica, julio de 1975) se aprobó una resolución que levanta la obligatoriedad de las sanciones y permite a todos los páses reanudar relaciones diplomáticas y comerciales con el gobierno de La Habana.

${ }^{30}$ "Great Decisions Program": Hearing before the Committee in Foreign Relations of the U.S. Senate. Government Printing Office, Washington, D.C., 1974 , p. 15 ,

"Ver "El Mercurio", 9 de mayo de 1975, p. 7.

${ }^{82}$ Ver Martin C. Needler, "Détente: Impetus for Change in Latin America", Journal of International Affairs, vol. 28, № 2, 1974.
} 
mantenga insatisfechos a un número considerable de países en vías de desarrollo, simultáneamente estará preparando el camino para su propio cuestionamiento y eventual disolución.

\section{OBSERVAGIONES FINALES}

a) ¿Ha habido realmente un proceso de distensión internacional?

$\mathrm{Si}$ consideramos los diversos tratados de Alemania Federal con Moscú y Varsovia, el acuerdo cuatripartito sobre Berlín, las negociaciones entre la República Federal Alemana y la República Democrática Alemana, los acuerdos soviético-norteamericanos SALT, las negociaciones en torno a la Conferencia sobre Seguridad y Cooperación Europeas, las reuniones en la cumbre entre EE. UU. y la URSS y, finalmente, el mejoramiento de relaciones entre EE. UU. y la República Popular China, y entre esta última y Japón, tendríamos que concluir afirmando que, efectivamente, ha existido un proceso de distensión o détente.

Especialmente significativos han sido los doce "Principios Básicos de las Relaciones entre los EE. UU. y la URSS", uno de los documentos más importantes firmados en la reunión de Moscú de 1972. Según el ex presidente Nixon los principales puntos del acuerdo eran los siguientes ${ }^{33}$ :

1) Ambas partes harán todo lo posible para evitar las conflagraciones militares y para prevenir el estallido de una guerra nuclear.

2) Ambas partes siempre ejercerán moderación en sus relaciones mutuas y estarán preparadas a negociar y arreglar sus diferencias por medios pacíficos. Las discusiones y negociaciones sobre los asuntos pendientes serán conducidas en un espíritu de reciprocidad, acomodación mutua y beneficio mutuo.

3) Las dos partes reconocen que cualquier esfuerzo para obtener ventajas unilaterales a expensas del otro, directa o indirectamente, es inconsistente con estos objetivos.

4) Ambas partes no reclaman para sí mismas ningún derecho especial o ventaja en los asuntos internacionales.

Según los norteamericanos, el principio general más importante sería el siguiente:

${ }^{33}$ Richard Nixon, "U. S. Foreign Policy for the 1970's", U. S. Government Printing Office, Washington, D.C., 1973, pp. 37-38. 
"Las partes procederán partiendo de la determinación común de que no existe alternativa que conducir sus relaciones mutuas sobre la base de la coexistencia pacífica. Las diferencias en ideología y en sistemas sociales entre EE. UU. y la URSS no son obstáculos para el desarrollo bilateral de relaciones normales basadas en los principios de la soberanía, la igualdad, la no-intervención en los asuntos internos y la ventaja mutua". ${ }^{34}$

Otro documento de suma importancia es el denominado "Comunicado de Shangai", firmado entre Nixon y Chou En-lai al término de la histórica visita que aquél hiciera a la República Popular China.

En aquella ocasión los dirigentes de EE. UU. y China pasaron revista a la situación internacional y expusieron sus respectivas posiciones y actitudes. En términos generales, ambas partes declararon lo siguiente ${ }^{95}$ :

1) El avance hacia la normalización de las relaciones entre China y los EE. UU. es conforme a los intereses de todos los países.

2) Ambas partes desean reducir el peligro de conflicto militar internacional.

3) Ninguna de las dos partes debe buscar la hegemonia en la región de Asia y del Pacífico y cada parte se opone a los esfuerzos de cualquier otro país o grupo por establecer tal hegemonía.

4) Ninguna de las dos partes está dispuesta a negociar en nombre de cualquier tercera parte o a concertar con la otra acuerdos o entendimientos dirigidos contra otros Estados.

5) Ambas partes son de la opinión de que estaría en contra de los intereses de los pueblos del mundo el que cualquier país grande se coluda con otro país grande contra los demás países, o que los países grandes dividan el mundo en esferas de intereses; y

6) Las dos partes toman el comercio bilateral como otro aspecto del que se puede derivar un beneficio mutuo, y convienen en que las relaciones económicas basadas en la igualdad y beneficio mutuo corresponden a los intereses de los dos pueblos.

b) ¿Cuáles han sido las consecuencias principales de la détente en el sistema internacional, en general, y en la estructura de alianzas, en particular?

aIbid., p. 37.

"Comunicado Conjunto Chino-Estadounidense", Ediciones en Lenguas Extranjeras, Pekín, 1972. 
En primer lugar, se podría afirmar que ha emergido una nueva "era de negociación" como consecuencia de la cooperación dentro del triángulo Moscú-Pekín-Washington. No obstante, por otro lado, se puede observar un deterioro en un segundo triángulo compuesto por EE. UU., Europa Occidental y Japón. En beneficio del proceso de distensión global, el profesor Robert L. Pfaltzgraff propone mejorar los vínculos entre los integrantes del segundo triángulo. En efecto, el autor señala que tanto a Moscú como a Pekín les interesa el mejoramiento de las relaciones entre EE. UU., Europa Occidental y Japón. Por ejemplo, la Unión Soviética y China verían con mucha preocupación el hecho de que Japón desarrollase su propio poderío nuclear, o si se aliase con una de las potencias socialistas a expensas de la otra. El profesor Pfaltzgraff sugiere que, para el establecimiento de una nueva estructura de paz mundial, "se debe otorgar primera prioridad a las relaciones en el triángulo EE. UU.-EuropaJapón. Aquí yacería la clave para la evolución de una nueva rela. ción con ambos, Moscú y Pekín..." Por lo tanto, la preservación del triángulo EE. UU.-Europa-Japón sería un prerrequisito esencial para el continuo mejoramiento de las relaciones entre EE. UU., la Unión Soviética y la República Popular China. ${ }^{36}$

En segundo término, el proceso mismo de distensión ha implicado un mayor margen de opciones para las potencias medianas y paises en desarrollo. De allí que algunas potencias medianas y pequeñas han sabido usar la détente para reafirmar su soberanía e independencia respecto de sus aliados mayores. Como consecuencia, el propio Kissinger ha sido duramente criticado por su preocupación excesiva por la diplomacia a nivel de super-potencias. El profesor Stanley Hoffmann de la Universidad de Harvard, sostiene que si bien Kissinger ha logrado sobrepasar la vieja concepción de guerra fría, ha cometido el error de pensar que bastaba con controlar la relación pentagonal para que todo lo demás se arreglase automáticamente. "El problema es que las dos super-potencias controlan cada día menos la política mundial... A través de todo el mundo se observa una reafirmación de las potencias pequeñas... No hemos sido capaces de controlar los acontecimientos en Tailandia o Portugal. $\mathrm{Ni}$ pudimos obligar a Israel a otorgar ciertas concesiones". "La única solución -según Hoffmann- es que los EE. UU. probablemente deberán aceptar un grado considerable de descentralización de la política exterior. ${ }^{37}$

Por otra parte, quizás este tipo de "descentralización" pueda con-

\footnotetext{
${ }^{80}$ Robert L. Pfaltzgraff, "Multipolarity, Alliances, and U. S. Soviet-Chinese Relations", Orbis, Fall 1973, Ne 3, pp. 723-724, 727-728 y 735-736.

${ }^{37}$ Stanley Hoffmann, citado en "Time", April 7, 1975, p. 29.
} 
tribuir al fortalecimiento de nacionalismos constructivos y regionalismos integradores que, en último término, se transforman en elementos estabilizadores del sistema internacional.

c) Por último, desde un punto de vista crítico de la détente cabe formularse varias interrogantes.

En primer lugar, es preciso considerar que la transición de un mundo bipolar a otro multipolar no necesariamente elimina la posibilidad de conflictos, por cuanto con la détente está abierta la posibilidad de "guerras limitadas en el plano económico, político y militar". A su vez existe el riesgo que estas conflagraciones acumuladas puedan "escalar" hasta niveles inaceptables para el sistema de equilibrio de poder. Es decir, a mayor cantidad de conflictos limitados en áreas secundarias, menor será la cantidad de zonas de entendimiento. ${ }^{38}$

En segundo término, esta nueva forma de entendimiento podría interpretarse como una simple estrategia destinada a preservar una situación de condominio. En otras palabras, la distensión podría conceptualizarse -al decir del profesor Robert Osgood- como "una estrategia para mantener un sistema internacional en el cual EE. UU. pueda retener su influencia global a un reducido nivel de esfuerzo y a un limitado riesgo de enfrentamiento armado". ${ }^{30}$

Finalmente, si bien la détente no es garantía contra la guerra total, parece que por parte de las potencias nucleares persiste el interés por continuar con el proceso, precisamente, con el objeto de impedir una conflagración nuclear. De allí que el secretario de Estado norteamericano Henry Kissinger haya expresado que, aunque subsiste una "hostilidad ideológica" entre Washington y Moscú, puede haber cooperación en "esferas limitadas", sobre todo en la "prevención de la guerra nuclear". 40

Por lo tanto, a pesar de las múltiples diferencias ideológicas y estratégicas de los diversos participantes en el proceso de distensión, parece subsistir un objetivo central: reducir -o si es posible eliminar- el peligro de una conflagración nuclear.

De cualquier manera, el análisis de un proceso tan complejo como el de la distensión aparece como un verdadero desafío para aquellos que la examinan, desde un punto de vista académico, y para quienes la implementan, desde un punto de vista práctico.

${ }^{38}$ Como ejemplo de un área conflictiva secundaria, ver las observaciones del profesor Morgenthau en relación a la "Enmienda Jackson" en Détente, op. cit., pp. 137-144.

${ }^{80}$ Robert E. Osgood, "The Consequences of Detente", SAIS Reviev, Winter 1973, vol. 17, No 2 .

${ }^{\star 0}$ Henry Kissinger, citado por agencia U.P.I. "El Mercurio", 30 de abril de 1975 , p. 9. 
BIBLIOGRAFA BASICA SOBRE EL TEMA DE LA "DÉTENTE"

ARTfCULOS $X$ LIBROS

Aubert de la Rue, Philippe - Les Relations Economiques entre l'Europe de l'est et l'Europe de l'ouest, Politique Etrangdre, 1973.

Bell, Coral - Middle East: Crisis Management during Détente, International Affairs (Chatham House), October 1974, No 4.

Brosio, Manlio - ¿Will NATO survive Détente?, The World Today (Chatham House), December 1972.

Buchan, Alastair - \&A World Restored?, Foreign Affairs, July 1972.

Carter, Barry - ¿What next in arms Control?, Orbis, Spring 1973, No 1.

Clemens, Walter C. - Nicholas II to SALT II: Change and Continuity in East-West Diplomacy, International Affairs (Chatham House), July 1973.

Cobb, Tyrus W. (Mayor) - Durabilidad de una Détente, Military Review, abril 1974, NQ 4.

Coffey, J. I. - Arms Control and the Military Balance in Europe, Orbis, Spring 1978 , No 1.

Chossudowsky, Evgeny - Genoa Revisited: Russia and Coexistence, Foreign Affairs, April 1972.

Dean, Robert W. - Bonn-Prague Relations: The politics of Reconciliation, The World Today (Chatham House), April 1973.

Dil, Shaheen - The Bangladesh Crisis and East-West Détente, SAIS Review, Winter 1973, vol. 17, No 2.

Dougherty, James E. - The Soviet Union and Arms Control, ORBIS, Fall 1973, No 3.

Fairbanks, John K. - The New China and the American Connection, Foreign Affairs, October 1972.

Figueroa, Francisco José - La Distension Europea: Causas, Efectos, Revista Estrategia, mayo-junio 1978, No 22.

Finley, David D. - Relaciones Soviético-Americanas en la Década de 1970, Mi. litary Review, junio 1973, No 6.

Gamson, William A. y Modigliani, A. - Untangling the Cold War, Little, Brown \& Co., Boston, 1971.

Gardner, Richard N. - The Hard Road to World Order, Foreign Affairs, April 1974.

Gati, Charles - East Central Europe: Touchstone for Détente, Journal of International Affairs, No 2, 1974.

Godoy, Horacio H. - Los acuerdos entre los EE. UU. y la URSS, Revista Estudios Internacionales, oct.-dic. 1974, No 28.

González, Idward - The United States and Castro: Breaking the Deadlock, Foreign Affairs, July 1972.

Hadick, Laszlo - The process of Détente in Europe, Orbis, Winter 1970, No 4.

Halpern, A. M. - The politics of Sino-Japanese Normalization, SAIS Review, Winter 1973, vol. 17, No 2.

Hassner, Pierre - Les contradictions de la détente: faux dilemmes et vrais problèmes, Defense Nationale, Juin 1974.

Hoffmann, Stanley - Oscillation and Innovation in American Foreign Policy, SAIS Review, Summer 1973, Weighing the Balance of Power, Foreign Affairs, July 1972. 
Heraldo Muñoz y Walter Sanchez / La Détento $\mathrm{g}$ el Sistema Intoraacional

Johnson, Chalmers - How China and Japan see Each Other, Foreign Affairs, July 1972.

Kaiser, Karl - Una nueva politica oriental en Alemania, Revista Estudios Internacionales, enero-marzo 1969, No 4.

Kennan, George F. - After the Cold War: American Foreign Policy in the 1970's, Foreign Affairs, October 1972.

Kielmansegg, Comte J. E. - Les problèmes de la détente militaire et de la re. duction des forces en Europe Centrale, Politique Etrangère, No 3, 1974.

Kinter, William R. - The U.S. and the URSS: Conflict and Cooperation, Orbis, Fall 1973, No 3 .

Koh, B. C. - The Korean Workers' Party and Détente, Journal of International Affairs, No 2, 1974.

Lagos, Gustavo - Vigencia de la Détente o Epitafio para la Humanidad, Revista Mensaje, octubre 1974, No 238.

Leonhard, Wolfgang - Domestic Politcs of the New Soviet Foreign Policy, Foreign Affairs, October 1973.

von Lohausen, H. Freiherr J. (general) - Estrategia de la Détente, Military Review, mayo 1974, No 5.

Needler, Martin C. - Détente: Impetus for Change in Latin America, Journal of International Affairs, No 2, 1974.

Osgood, Robert ๘. - The Consequences of Détente, SAIS Review, Winter 1973. vol. $17, N^{\circ} 2$.

Parkinson, F. - Latin American Foreign Policies in the Era of Détente, International Affairs, July 1974, No 3.

Pfaltzgraff, Jr., Robert L. - Multipolarity, Alliances and U.S.-Soviet-Chinese Relations, Orbis, Fall 1973, No 3.

Pfaltzgraff, Jr., Robert L. y Kintner, William R. - Assesing the Moscow SALT Agreements, Orbis, Summer 1972, No 2.

Pierre, Andrew J. - L'accord SALT et ses conséquences pour l'Europe (I), Politique Etrangère, N\& 3, 1972.

Ranger, Robin - Canadian Foreign Policy in an Era of Super-Power Détente, The World Today (Chatham House), December 1972.

Rhee, T. C. - Peking and Washington in a New Balance of Poker, Orbis, Spring 1974, No 1.

Rhee, T. C. - Implications of the Sino-American Détente, Orbis, Summer 1972, No 2.

Sánchez Sorondo, Marcelo - Argentina ante la Coexistencia Ruso-Norteamericana, Revista Estrategia, nov. 72-feb. 73, Nos. 19-20.

Scoville, Hetbert - Beyond SALT One, Foreign Affairs, April 1972.

Schlesinger, A. M. - Origins of the Cold War, Foreign Affairs, October 1967.

Schutze Walter - L'accord SALT et ses consequences pour l'Europe (II), Politique Etrangère, No 3, 1972.

Shepard, J. C. (T. Coronel) y otros - La posibilidad de una Détente, Military Review, julio 1974, No 7 .

Schwiwsor, Naomi - Détence and Europe, SAIS Review, Winter 1973, vol. 17, No 2.

Shulman, Marshall D. - Towards a Weslern Philosophy of Coexistence, Foreign Affairs, October 1973.

Slovinski, Martin J. (coronel) - Facetas de SALT, Military Review, enero 1974, No 1.

Stone, Jeremy J. - When and How to use SALT, Foreign Affairs, January 1970.

Swanson, Roger F. - East-West Détente and the US-Canadian relationship, SAIS Revicw, Spring 1972. 
ESTUDIOS INTERNACIONALES

Thee, Marek - US-Chinese rapprochement and Vietnam, Journal of Peace Research, NQ 1, 1972.

Toinet, Marie France - La nouvelle stratégie américaine et SALT II, Défenses Nationales, Juin 1974.

Tiersky, Ronald - The French Communist Party and Détente, Journal of International Affairs, No 2, 1974.

Valenta, Jiri - Soviet Foreign Policy and the Moscow Summit of 1972, SAIS Review, Winter 1973, vol. 17, No 2.

Vélez, Lisandro - Reanudación de las relaciones entre Japón y China: Importante etapa de la coexistencia pacifica, Revista Estrategia, sept.-octubre $1972, \mathrm{~N} 218$.

Vcrnant, Jacques - Reflexions sur la "délente", Politique Etrangère, Nos. 4-5, 1974.

Whetten, Lawrence L. - Appraising the Ostpolitik, Orbis, Fall 1971, No 3.

Wilson, Duncan - Anglo-Soviet Relations: the effect of Ideas on Reality, International Affairs, July 1974, No 3.

Wilson, Edward T. - Some Perspectives on the Recent Expansion in US-Soviet Commercial Relations, SAIS Review, Winter 1973, vol. 17, No 2.

Windsor, Philip - The Boundaries of Detente, The World Today (Chatham House), June 1969.

Zellentin, Gerda - Institutions for Détente and Cooperation, The World Today (Chatham House), January 1973.

DOCUMENTOS

"Comunicado Conjunto Chino-Estadounidense", Ediciones en Lenguas Extranjeras, Pekín, I972.

"Détente", Hearings before the Subcommittee on Europe of the Committee on Foreign Affairs, House of Representatives, U. S. Government Printing Office, Washington, D.C., I974.

"Discurso del Presidente Ford sobre Relaciones Extcriores", texto oficial, Servicio de Cultura y Prensa de la Embajada de EE. UU., Santiago, 1975.

"Evaluación de la Détente de Henry Kissinger", texto oficial, Servicio de Cultura y Prensa de la Embajada de EE. UU., Santiago, I975.

"Great Decisions Program", Hearing before the Committee on Foreign Relations of the United States Senate, U. S. Government Printing Office, Washington, D.C., 1974.

"Surgimiento del Tercer Mundo y Declinación del Hegemonismo", corresponsal de Sinjua, Boletín Informativo de la Embajada de la República Popular China, Santiago, 1975.

"U. S. Foreign Policy for the 1970's: a new Strategy for Peace", Richard $M$. Nixon, U. S. Government Printing Office, Washington, D.C., 1970.

"U. S. Foreign Policy for the 1790's: Shaping a Durable Peace", Richard M. Nixon, U. S. Government Printing Office, Washington, D.C., 1973.

"Détente", Hearings before the Committee on Foreign Relations U. S., Senate U. S. Government Printing Office, Washington, D.C., 1975. 